\title{
La prensa profesional como fuente de información historiográfica en la Historia de la Enfermería
} The professional press as a source of historiographical information on the History of Nursing

\section{A imprensa profissional como uma fonte de informação historiográfica sobre a História da Enfermagem}

\author{
Antonio Jesús Marín Paz
}

Diplomado en Enfermería. Máster Universitario en Innovación e Investigación en Cuidados de Salud.

\begin{abstract}
Cómo citar este artículo en edición digital: Marín Paz, A.J. (2017). La prensa profesional como fuente de información historiográfica en la Historia de la Enfermería. Cultura de los Cuidados (Edición digital), 21(47).

Recuperado de http://dx.doi.org/10.14198/cuid.2017.47.07

Correspondencia: C/ Arquitecto Torcuato Cayón no $12^{\circ}$ A. 11100. San Fernando (Cádiz).

Correo electrónico: antoniomarinpaz@hotmail.com

Recibido: 28/04/2016; Aceptado: 10/09/2016
\end{abstract}

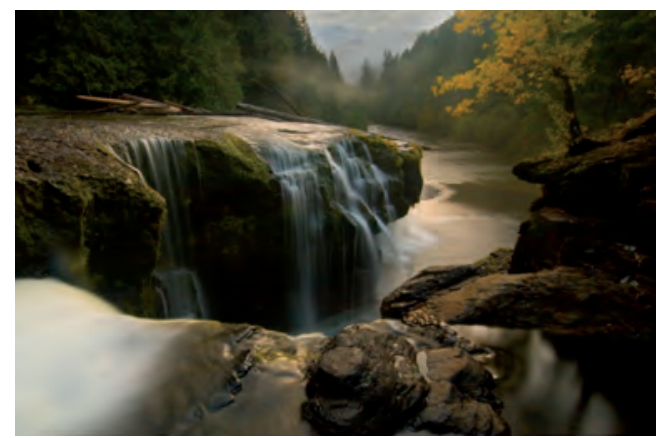

\section{ABSTRACT}

The nurse and previous auxiliary professions press, is a valuable source for the study of evolution developed and its interrelationship between different health disciplines. Knowing and studying serials publications in this sector allow us to approach reality and professional identity, educational and current which hit.

In this article is proposed a metodological guide for finding this kind of source of historiographical information and a model for the historical study of the nurse professional press based on the latest developments in the historiographical methodology; supported on separate analysis of the structure of the press by the precepts of Jaques Kayser and its contents by themes through heuristic, hermeneutic and historical methods in the interpretation of texts.

Keywords: Nurses, midwives, ministrantes, practicantes, press.

\section{RESUMO}

A imprensa profissional enfermeiro e suas profissões antecedentes de saúde é uma fonte valiosa para o estudo da evolução que desenvolveu e sua inter-relação entre as diferentes disciplinas da saúde. Conhecer e estudar folhetins nesse setor nos permite abordar a realidade e identidade profissional, educacional e tópica que atingiu.

Neste artigo tenciona um guia metodológico para encontrar este tipo de fonte de informação historiográfica e um modelo para o estudo histórico da imprensa profissional enfermeiro com base nos mais recentes desenvolvimentos na metodologia historiográfica; suportado pela análise separada da estructura da imprensa os preceitos de Jaques Kayser e seu conteúdo por temas através de heurística, 
hermenêutica e método histórico na interpretação de textos.

Palavras Chave: Enfermeiros, Parteiras, Minitrantes, Practicantes, Imprensa.

\section{RESUMEN}

La prensa profesional enfermera y de sus antecedentes profesiones auxiliares, constituye una fuente valiosa para el estudio de la evolución que desarrollaron y su interrelación entre las diferentes disciplinas sanitarias. Conocer y estudiar las publicaciones seriadas de este sector nos permite acercarnos a la realidad e identidad profesional, formativa y de actualidad por la que atravesaban. En el presente artículo se propone una guía metodológica para la búsqueda de este tipo de fuente de información historiográfica y un modelo para el estudio histórico de la prensa profesional enfermera basado a los últimos avances en la metodología historiográfica; apoyado en el análisis por separado de la estructura de la prensa mediante los preceptos de Jaques Kayser y de su contenido por ejes temáticos a través los métodos heurístico, hermenéutico e histórico en la interpretación de textos.

Palabras clave: Enfermeras, matronas, ministrantes, practicantes, prensa.

\section{INTRODUCCIÓN}

La Historia de la Enfermería se encuentra en un periodo de auge desde la incorporación de la titulación a la Universidad. A través de este factor, hemos comprobado una evolución en los últimos 35 años, tanto en la temática como los contenidos metodológicos realizados por tantos investigadores de la enfermería (Hernández Martín, 2008).

En la actualidad, el historiador tiene a su disposición un elevado número de fuentes de información historiográfica de diversa índole, desde los tradicionales archivos históricos hasta las entrevistas en el ámbito de la historia oral. Así, pues, el investigador puede realizar una selección de sus fuentes en función de su fiabilidad y adecuación a la problemática seleccionada a resolver (Siles González, 2011). La prensa constituye una fuente de información historiográfica de indudable valor en la Historia Contemporánea (Barrera del Barrio, 2000), aportando una mayor cantidad de información escrita que otras, además de los múltiples periódicos y revistas que coexistieron en los mismos años y que diferían en sus posturas políticas e ideológicas; desde las favorables al gobierno hasta las minorías o clases sociales menos pudientes (Tuñón de Lara, 2009). No podemos olvidar el hecho de que suele constar de información relevante y variada en función de la época que se esté consultando. Por ejemplo, hay que tener muy presente que la prensa durante la Dictadura de Primo de Rivera era revisada y validada por el comité de censura.

Así, pues, información e ideología suelen ser características interrelacionadas que es preciso discernir en cualquier prensa. En las secciones de opinión, a falta de poder recoger fuentes orales debido a su lejanía en el tiempo, se puede observar el punto de vista $y$ la mentalidad de las personas que escribieron, pudiendo abarcar desde una persona célebre hasta personas o profesionales poco conocidos (Valle Racero, 2000).

La prensa puede clasificarse en función del público al que se dirija. En nuestro caso, es destacable la división en prensa general y prensa profesional. La prensa general engloba todo tipo de temáticas y diferentes formatos; por ello suelen tener una periodicidad corta (en muchos casos, son diarios). Ello conforma un tipo de fuente de información historiográ- 
fica valiosa para el estudio de la sociedad de los años estudiados y se desprende que a partir de ella podríamos conocer la visión que tiene la sociedad con respecto a la sanidad (Heierle Valero, 2009); y más específicamente, a las ramas sanitarias auxiliares y de enfermería en función de la época (Rodríguez García, 1994) (Herrera Rodríguez, 2008). Dada la gran cantidad de información, la ardua tarea de recopilar noticias y artículos de opinión sobre la profesión requiere invertir mucho tiempo y paciencia, pero tiene la compensación de que este tipo de prensa nos ofrece una visión diferente de los profesionales y de la población a la que atendían. Aunque normalmente dedican mayor información a las figuras médicas notables, en ocasiones encontramos apuntes sobre practicantes, matronas y enfermeras; ya sea sobre sus laborales asistenciales, colegiales o incluso de organización y convocatorias de enseñanza de sus respectivas carreras (Lasarte Calderay, 2000).

En cambio, la prensa profesional enfermera (haciéndose extensible a la prensa profesional española de los ministrantes, practicantes, matronas y A.T.S.), editada por y para los profesionales, presenta temáticas específicas de la profesión y del entorno en el que se desenvuelven. Su periodicidad es mayor (con frecuencia quincenal, mensual, trimestral o semestral) y contiene información de primer orden para identificar la evolución de la identidad y memoria profesional enfermera de los últimos siglos (Bernabeu Mestre y Gascón Pérez, 1995); además de conocer la posición de la profesión en la sociedad, desde el ámbito local hasta el internacional (Herrera Rodríguez, 1995) (Siles González, 2004). A diferencia de la prensa general, el escaso número de ejemplares puede provocar vacíos temporales y de contenido en nuestro estudio (máxime si se carece de la se- rie completa de la prensa consultada, por esto aparte de los archivos y bibliotecas, que posteriormente comentaremos, hay que tener muy presente la localización de números de estas revistas en archivos particulares).

Por lo tanto, nuestro objetivo es presentar una guía metodológica y un modelo para el estudio de la prensa histórica profesional enfermera, así como sus diversas aplicaciones para la mejora del conocimiento en la Historia de la Enfermería desde mediados del siglo XIX.

\section{DESARROLLO DEL TEMA}

\section{Una guía metodológica para el estudio de la prensa profesional.}

Como toda investigación basada en el método heurístico, el primer paso imprescindible es la delimitación de nuestro campo de estudio a través del conocimiento del estado actual del tema. No sólo hay que conocer la estructura histórica profesional, también es imprescindible conocer y relacionar la situación histórica en el que se desenvuelven, de tal manera que podamos explicar los factores que intervienen, acercándonos a su vez, a la historia total que en su día reivindicaron Lucien Febvre y Marc Bloch (Itayra Padilla, Borenstein y Guedes, 2007). Una vez realizada la revisión bibliográfica, es posible que nos preguntemos ciertos aspectos relacionados con la prensa profesional o bien detectemos vacíos temporales y de contenido que se podrían abordar (Santo Tomás Pérez, 2006). Dependiendo de la cuestión que queramos tratar, seleccionaremos el estudio de prensa general o prensa profesional enfermera específica: o ambas si es necesario.

La localización de fuentes es variable. Por un lado, afortunadamente se conservan numerosos ejemplares de las revistas más recientes, mientras que por otro, a medida que retrocedamos en el tiempo, será más difícil encontrar 
la totalidad de ejemplares de la prensa que queramos investigar. Debemos tomar nota en que se trata de un tipo de producción periodística que ha sido poco valorada hasta su incipiente estudio hace pocas décadas (Herrera Rodríguez y Lasarte Calderay, 1996). Tampoco debemos desdeñar la labor de consulta de catálogos bibliográficos y estudios bibliométricos para tener constancia de la existencia de una determinada publicación (Martínez Sánchez, 1901) (López Piñero y López Terrada, 1991) (Álvarez Nebreda, 2010).

Actualmente, la prensa, ya sea general o profesional, se pueden encontrar en los siguientes emplazamientos en España, pero en algunos casos son extensibles a otros países:

- Biblioteca Nacional de España (Madrid). La biblioteca más importante del país, posee un extenso catálogo de prensa general y profesional enfermera en su sección de "Prensa y revistas". Disponen de ejemplares físicos y microfilmados, aunque actualmente existen restricciones para el acceso a las publicaciones anteriores a 1958 (dependiendo del carné que posea el usuario).

- Colegios Oficiales de Enfermería. Sin duda, los Colegios de Enfermería, motor de la profesión durante muchos años, suelen disponer de la mayoría de los ejemplares de las revistas provinciales. Especialmente relevante es el caso del Colegio Oficial de Enfermería de Madrid (CODEM) y su prolífica hemeroteca, con más de 50 publicaciones diferentes de prensa profesional enfermera. Hay que destacar que esta institución está haciendo un esfuerzo digitalizando libros y revistas para su disponibilidad en la red, entre los cuales, podemos mencionar la revista Medicina y Cirugía Auxiliar (1941-1978).

- Bibliotecas de las universidades. La prensa histórica profesional enfermera, en especial del ámbito provincial, pueden encontrarse en los diferentes depósitos de las bibliotecas de las universidades, como es el ejemplo de la Universidad de Zaragoza con la revista El Practicante (1885). El principal inconveniente estriba en su acceso: es de relativa facilidad para docentes y alumnos de la propia Universidad y más dificultoso en caso de un investigador externo. Debemos subrayar el caso de la Biblioteca de Ciencias de la Salud (Universidad de Cádiz) que custodia la donación por José Eduardo Lasarte Calderay de varios centenares de números fotocopiados de las siguientes revistas: El Practicante Gaditano (1916-1939), El Practicante Sevillano (1923-1936), La Federación Sanitaria (1925-1928), El Auxiliar Médico de Córdoba (1935-1936) y El Practicante Almeriense (1919-1936).

- Instituciones docentes privadas o religiosas. Buena parte de la profesión enfermera ha ido ligada a través del tiempo con instituciones de carácter privado o religioso, por lo que en ocasiones estos centros conservan determinadas revistas específicas de enfermería. La institución Salus Infirmorum es un ejemplo de ello con la revista ¡Firmes! (1953-1965).

- Bibliotecas públicas y hemerotecas. En estos centros podremos encontrar en su catálogo, revistas de carácter provincial. Especialmente importante este aspecto, porque a menudo conservan los únicos ejemplares que puedan existir de determinadas revistas. En general, estas instituciones tienen gran variedad de prensa general accesible al público. Podemos citar algunos ejemplos como la Biblioteca Pública Provincial de Cádiz y la Hemeroteca Municipal de Madrid.

- Biblioteca Virtual de Prensa Histórica. En este portal disponible a través de la red informática mundial, podemos encontrar ejemplares de varias revistas de practicantes 
debidamente digitalizadas para su consulta. Un ejemplo de ello es El Practicante Español (1899).

- Archivos históricos. En ocasiones, los diferentes archivos históricos del país pueden disponer de prensa histórica, principalmente de carácter local, aunque es posible encontrar en sus cajas algún ejemplar de prensa profesional enfermera si contiene información relevante de la población donde se emplace el archivo histórico.

- Archivos particulares o privados. Determinadas personas por diferentes motivos (coleccionismo, herencias, interés en la temática..., etc) pueden poseer ejemplares de revistas enfermeras en sus respectivos hogares y que tienen predisposición a ceder temporalmente sus archivos para su estudio. Podemos citar la biblioteca particular de Luis García Jiménez y su colección de ejemplares de El Practicante Gaditano del periodo 1930 a 1934.

Debemos considerar otro aspecto: el soporte en el que se encuentren dichas fuentes. Por regla general, se encuentran en formato físico y a veces no se permite la consulta debido a su precario estado de conservación. Como consecuencia de los avances de la tecnología óptica en las últimas décadas, es posible que se dispongan de ejemplares microfilmados o en microfichas, por lo que se da la oportunidad al investigador de poder realizar una copia digital (si disponen de soporte informático para ello). Afortunadamente, a partir de la publicación de la Ley 11/2007, de 22 de junio, dedicada al acceso electrónico de los ciudadanos a los Servicios Públicos, poco a poco las diferentes bibliotecas, hemerotecas y archivos históricos se encuentran en un proceso de digitalización de la prensa en consonancia con los presupuestos que disponen, no sólo para favorecer su acceso (ya sea en el propio centro o a través de bibliotecas virtuales, tanto nacionales como internacionales), sino también para la conservación de la prensa física custodiada. En la actualidad podemos encontrar una serie de dificultades en el acceso de determinadas prensa profesional enfermera, que aún sigue sin ser digitalizada, como es el caso de las diferentes revistas históricas de la Cruz Roja.

A modo de ejemplo, en el estudio realizado se puede adjuntar una tabla sencilla con los números consultados, para que cualquier lector que necesite conocer o estudiar el tema, sepa en todo momento los contenidos que han sido sometidos a juicio crítico por parte del investigador. En las filas podemos emplazar los años, en las columnas los meses (o bien numerados) $\mathrm{y}$, por último, en las restantes celdas, insertar los números consultados en función de su fecha (un color para cada revista si se han consultado varias; y en caso de ser una revista con una nomenclatura de volúmenes, numerar el volumen y entre paréntesis insertar el número; en consonancia con el estilo APA). (Tablas 1 y 2 ).

Tabla 1. Ejemplares de "El Practicante Valenciano" en el CODEM.

\begin{tabular}{|c|c|c|c|c|c|c|c|c|c|c|c|c|}
\hline FECHA & ENE & FEB & MAR & ABR & MAY & JUN & JUL & AGO & SEP & OCT & NOV & DIC \\
\hline 1905 & & & & & & 15 & 16 & 17 & 18 & 19 & 20 & 21 \\
\hline 1906 & 22 & 23 & & & & & & & & & & \\
\hline
\end{tabular}


Tabla 2. Ejemplares de "Caridad, Ciencia y Arte: Boletín de las enfermeras y A.T.S. de España" de la $1^{a}$ época (1963-1971) y de la $2^{a}$ época (1972-1977) en el CODEM.

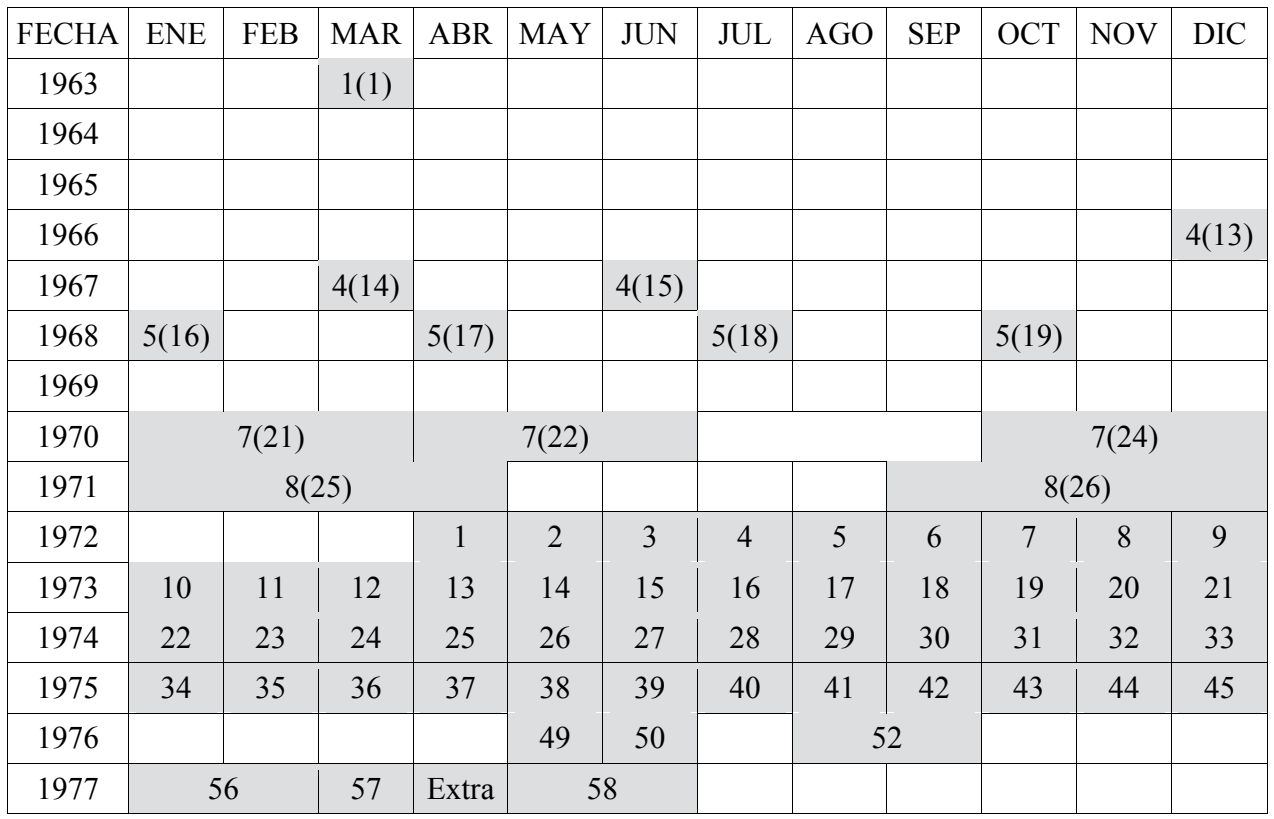

Fuente: CODEM (2013)

En función de las preguntas que nos formulemos, debemos abordar la prensa de una manera $\mathrm{u}$ otra. Si nuestra intención es tratar un determinado acontecimiento de la profesión, lo más factible sería consultar diferentes fuentes periodísticas, tanto general como prensa profesional, de la época a tratar (Rodríguez García, 1994). En este sentido debemos consultar una extensa bibliografía de apoyo como contraste para la información que analicemos.

Si nuestro objetivo está orientado a seguir la evolución de una determinada publicación a lo largo del tiempo para establecer relaciones con otras o bien analizar exhaustivamente una sección en concreto, deberíamos realizar una recopilación de todos los ejemplares que estén disponibles de una determinada publicación.

Independientemente de la estrategia que hayamos escogido, lo primero que debemos hacer es realizar una lectura inicial para familiarizarnos con la prensa consultada y tomando nota de los principales temas que se tratan junto con los diferentes colaboradores principales. Explicaremos a continuación cómo podría realizarse desde nuestro punto de vista un estudio de una determinada prensa profesional enfermera, basándonos en el método histórico en la interpretación de textos (Siles González, 2001b).

\section{Un modelo para el estudio de la prensa his- tórica profesional enfermera.}

Una vez localizada la documentación se debe proceder, además del método citado previamente, de acuerdo a las normas marcadas por autores como Sánchez Granjel (1967), Febvre (1974), Pierre Salmon (1978), David Romano (1983), Tuñón de Lara (1985), Thuillier y Tullard (1989) o Aróstegui (1995). 
Comenzaremos a revisar concienzudamente cada número. Cada revista, dependiendo de la época, puede variar en forma y contenido, aunque siempre podremos apreciar una serie de aspectos análogos. (Figura 1)

Figura 1. Temáticas para el estudio e interpretación de revistas profesionales (ministrantes, practicantes, matronas y enfermeras).

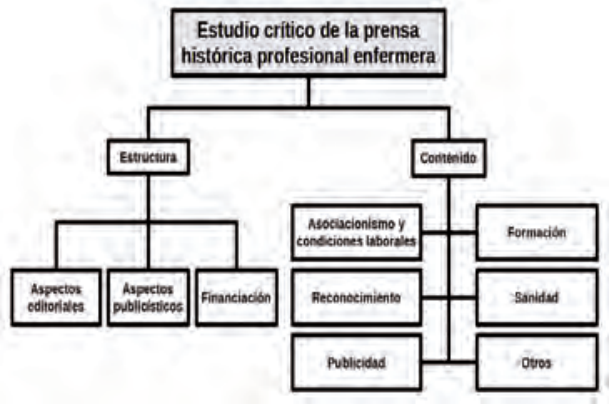

Fuente: Elaboración propia (2014).

\subsection{Estudio de la estructura:}

Es importante que el conocedor de la prensa profesional siga las normas marcadas por Jacques Kayser hace ya más de medio siglo; por ejemplo, en lo que se refiere al "registro de identificación" del periódico o revista. (Tabla 3).

Tabla 3. Principales aspectos de estructura a recoger en un estudio de prensa.

\begin{tabular}{|l|}
\hline REGISTRO DE IDENTIFICACIÓN \\
\hline Nombre del periódico o revista (anotar los \\
cambios en la denominación y las \\
respectivas fechas). \\
Lugar de la residencia de la Administración \\
y de la redacción, consignando \\
igualmente si se producen cambios. \\
Las indicaciones que se puede acompanar al \\
nombre. \\
La periodicidad. \\
El momento de la aparición. \\
Zona principal de difusión. \\
Ejemplares que compone cada tirada. \\
Fecha del primer y último número. \\
Precio. \\
Formato. \\
Número de páginas. \\
Nombre y dirección del impresor. \\
Lugar y conservación de las colecciones.
\end{tabular}

Lo primero que debemos observar es la portada, ya que a medida que nos acercamos al tiempo presente, la imagen de portada podría variar en cada número, constituyendo una fuente iconográfica valiosa de estudio propio si lo requiriese. La revisión del título, lema y el equipo de redacción pueden cambiar con el paso de los años y, por ello, conllevar a un cambio en la mentalidad y en los objetivos del periódico o de la revista. Conocer la tirada y, por ende saber el número exacto de ejemplares que se distribuían a los profesionales en la época estudiada, puede hacernos una idea de la extensión y de la importancia que tuvo una determinada publicación seriada.

Es muy importante para la memoria histórica de los profesionales sanitarios el dar a conocer en nuestro estudio, los nombres y apellidos de tanto del equipo de redacción como el de los principales colaboradores. Es una realidad comprobar que estamos más familiarizados con los nombres y apellidos de otras profesiones del pasado que de nuestros propios profesionales (ya sean ministrantes, practicantes, matronas, A.T.S. o enfermeras; como ya comentamos anteriormente).

Generalmente las revistas enfermeras se encuentran divididas en diferentes secciones, que pueden variar de una publicación a otra. Especialmente relevante son las secciones literarias, en las que podemos encontrar narraciones y poesías. Las fotos y figuras que aparecen pueden ser objeto de estudios iconográficos al igual que las portadas, principalmente en lo referente a retratos de profesionales, eventos o problemas históricos de mayor complejidad, como es el caso de las noticias y fotografías de El Practicante Gaditano sobre la huelga de sanitarios liderada por Fermín Aranda en 1919 en la ciudad de Jerez de la Frontera (Herrera Rodríguez y Cabrera Afonso, 2001). 
La prensa es destinada al consumo público, ya sea para la población general como para un reducido sector, y como tal, se vale, además del pago de los lectores (mediante pago directo o bien por suscripción), de la publicidad como método de financiación (García Hernández y Gálvez Toro, 2002a). Recuérdese que el establecimiento tipográfico (o imprenta) suele ser independiente de la redacción, además de que se necesitan vías de ingreso para los salarios del equipo de redacción. La publicidad puede ser de tipo general (establecimientos, servicios..., etc) y específica para la profesión (cursos, productos farmacéuticos..., etc).

\subsection{Estudio del contenido:}

La corriente ideológica de una publicación seriada obedece en muchos casos al contexto histórico en el que se desenvuelve: la prensa profesional enfermera no es una excepción. Sin embargo, presenta una serie de diferencias con respecto a la prensa generalista, recordando en todo momento los preceptos señalados por Jaques Kayser.

El estudio del contenido debe basarse en una lectura concienzuda y pormenorizada, basada en el método heurístico, como hemos indicado anteriormente. A través de la hermenéutica, se pretende establecer una base racional de las corrientes intelectuales en la que se engloban dichas fuentes de información historiográfica. El procedimiento requiere de un análisis documental en niveles de profundidad, desglosando cada idea aportada en el texto para establecer un esquema que nos permita averiguar las estructuras mentales y los condicionantes que influyeron en el proceso de escritura; y comprender el significado de las acciones humanas en la exposición del acontecimiento histórico (Aróstegui Sánchez, 1995). Por otra parte, la escritura de nuestro estudio debe alejarse del carácter positivista (basado en la historia-relato, reduciendo al historiador como mero consignador de datos sin crítica de los hechos) y acercarse a las nuevas tendencias de la historiografía (Hernández Sandoica, 2004) (Moral de Calatrava y Hernández Conesa, 2007). Con todo ello se podría conseguir un análisis del texto y una escritura crítica acorde al período estudiado.

A tenor de nuestra experiencia al estudiar las diferentes publicaciones, podemos distinguir cuatro ejes importantes de estudio referente a su contenido:

Eje I: asociacionismo, identidad profesional, luchas con otros sectores de la misma profesión $u$ otros profesionales sanitarios y las condiciones laborales. Este tema es tratado desde las primeras publicaciones hasta la actualidad. A menudo los ministrantes y practicantes denunciaban el intrusismo de las enfermeras, principalmente a partir del auge de la formación de las enfermeras en la Escuela de Enfermeras Santa Isabel de Hungría (Lasarte Calderay, 1993) (Rodríguez García, 1994) (Calvo Calvo, 2014). Las matronas a su vez acusaban a los practicantes de ejercer en partos en poblaciones pequeñas en las primeras décadas del siglo XX (García Martínez y Espina Gutiérrez, 1998-1999).

El intrusismo va evolucionando con el tiempo y la enfermería también miraba con recelo a otras ramas sanitarias, como es el caso de la década de 1950 con otras profesiones auxiliares sanitarias respecto a la luchas sociales y sus competencias (Córdoba Mariscal, 1994). Para este punto, es importante establecer quiénes son los profesionales implicados, la situación legislativa de la época estudiada y, por supuesto, la valoración de las distintas opiniones de las diferentes facciones. 
Otro tema importante a tratar en este apartado son las condiciones laborales de la profesión: no es lo mismo el trabajo de un practicante o matrona en el ámbito rural que la pertenencia al Cuerpo de Beneficencia de una capital de provincia a principios del siglo XX. A su vez, desde la propia Sociedad de Ministrantes, el tema recurrente de la protección social a través de seguros y montepíos nos aproxima a las dificultades económicas por las que atravesaba el propio profesional y sus consecuencias en los familiares más allegados (Ávila Olivares, 2014).

Una última cuestión de interés, es que la mayoría de las publicaciones pertenecen o han pertenecido a Colegios provinciales: no debemos desviar nuestra atención a los acontecimientos que se produzcan en ellos y que hayan sido plasmados en la revista a estudio.

Eje II: formación a través de cursos, titulaciones y manuales. Los profesionales de la enfermería históricamente han sabido plasmar el ideal de que, para mejorar el reconocimiento profesional en la sociedad, debían de ser útiles a ella, y para eso hacía falta perfeccionar su formación a través de la adquisición de nuevos conocimientos, habilidades o técnicas que repercutan en beneficio de la salud de la población. En ocasiones, las revistas enfermeras daban a conocer a sus lectores nuevos manuales o bien, mediante anuncios, se anunciaban diferentes cursos de perfeccionamiento o para adquirir una determinada titulación, entre los cuales se pueden citar las técnicas de cirugía menor recogidas en la sección científica de La Voz de los Ministrantes (1864) o bien los cursos de enseñanza de las asignaturas de la titulación de Practicante con autorización a la asistencia a los partos normales en El Practicante Valenciano (1905).
Eje III: reconocimiento de los profesionales en la sociedad y entre compañeros sanitarios. A través de la formación y a la aplicabilidad de dichos conocimientos, es posible que un determinado profesional de las diferentes ramas sanitarias auxiliares que existieron desde el siglo XIX, fuese premiado por haber realizado alguna obra de sumo interés, no sólo para la ciencia, si no para la contribución del desarrollo del progreso a las condiciones sanitarias de la población. A veces, la prensa profesional también realizaba un reconocimiento a un determinado profesional de otra rama sanitaria que, por alguna razón, se encuentre estrechamente relacionada con la profesión enfermera. Podemos citar la felicitación de El Eco de las Matronas (1898) al médico Dr. Andrés Martínez Vargas por su ingreso en la Sociedad de Pediatría de Moscú.

Eje IV: visión de los profesionales con respecto a la Sanidad de la época estudiada. Los profesionales de enfermería y de sus antiguas ramas, son testigos y, por supuesto, forman parte del motor que contribuye al desarrollo de la Sanidad. Por lo tanto, ofrecen un punto de vista detallado y más íntimo de la marcha del desarrollo de la Sanidad de un país; ya que normalmente han sido especialistas, por lo que ofrecen un punto de vista humanístico al campo de la salud. La prensa enfermera no es ajena a ello: no duda en realizar artículos de crítica o de opinión en las diferentes épocas en pro de mejorar las condiciones sanitarias de la población. La denuncia de los practicantes de Madrid a través de El Practicante Moderno (1903) sobre la clausura del hospital de epidemias del Cerro del Pimiento en época de epidemia de viruela, es un ejemplo de ello (Marín Paz, 2014). Por lo tanto, recoger estas opinio- 
nes nos brinda un acercamiento y una visión única de la Sanidad de los años estudiados.

Por supuesto, estos ejes son orientaciones para el análisis de contenido, pueden variar o bien añadir algún aspecto que sea particularmente novedoso en alguna publicación. Es de reseñar el cariz hispanoamericano que se caracterizaba la revista Enfermeras en 1950-1951 (Córdoba Mariscal y Herrera Rodríguez, 1992). Para el estudio del contenido de la prensa profesional, es muy útil como orientación la consulta previa de referencias sobre la historia de las fuentes periodísticas de las profesiones sanitarias (Riera Palmero, 1978), además de estudios precedentes que son de obligada lectura como los trabajos de Raúl Expósito González.

Una vez escogida la prensa a investigar, es muy importante delimitar su estudio en función de su periodo de publicación y del contenido a analizar, utilizando una base de datos o las tradicionales fichas bibliográficas que recomendaba a sus discípulos el profesor Luis Sánchez Granjel para registrar los cambios que se produzcan tanto en la estructura como en el contenido a lo largo de los diferentes números consultados. Con ello se pretende un mejor ordenamiento de las temáticas y de las ideas principales, además de brindarnos la posibilidad de realizar un análisis cuantitativo de las diferentes secciones de las revistas enfermeras.

Las conclusiones de cada estudio que en esta línea se realicen deben siempre estar referidas a los objetivos que se planteen en la introducción del trabajo. También debemos preguntarnos si a través de la revista o periódico que se plantee el historiador, se podrían llegar a conclusiones influyentes para la Historia de la Enfermería. Señalamos algunas sugerencias por si son de utilidad a los futuros investigadores: ¿qué hace diferente una publicación de otras? ¿Qué aportaciones originales hemos encontrado? ¿Existen contradicciones entre diferentes publicaciones? ¿Hemos detectado un desequilibrio de los contenidos a través de un análisis cuantitativo? ¿Cuál fue la visión de una población referente a la enfermería y sus predecesores? ¿Podemos analizar una época determinada de la enfermería a través de la prensa profesional y generalista?

\section{CONCLUSIONES. EL FUTURO DE LA CUESTIÓN}

El análisis de la prensa, tanto profesional como general, nos ofrece un amplio repertorio de posibilidades para mejorar el conocimiento histórico de la enfermería desde mediados del siglo XIX (Expósito González, 2013a). Comprende desde el estudio general de una determinada prensa histórica profesional enfermera que no haya sido sometida a estudios previos (y, por tanto, podría realizarse un análisis crítico de todo el contenido de los números en función del tiempo de publicación) hasta el estudio minucioso de una determinada sección de una sola publicación, aunque haya sido estudiada previamente en sus aspectos generales en anteriores estudios (Callejas Chagoyen y Miqueo, 2014).

El conocimiento sobre las relaciones de profesionales de otras ramas sanitarias con la profesión enfermera podría suponer una nueva línea de investigación en un futuro no muy lejano, como es el caso de la estrecha relación que mantienen los médicos tocólogos y las matronas en El Eco de las Matronas. Tampoco debemos olvidar la impresión de la propia población respecto a los profesionales a través de la prensa generalista, además de la participación de estos últimos en este tipo de prensa. Una observación sobre este punto es que, a partir de 1975, esta prensa ofrece múltiples posibilidades para cumplir con este objetivo. 
Por lo tanto, no se trata de un tipo de investigación aislada en la Historia de la Enfermería, es un tipo de fuente de información historiográfica que refleja de manera muy aproximada los sentimientos y la mentalidad de nuestros profesionales precedentes, y por tanto complementaria de otras fuentes, sobre todo para cuando no sea posible abarcar ese campo de conocimiento en caso de la lejanía en el tiempo de la prensa profesional seriada (Sanz Hernández, 1995).

La búsqueda de las diferentes publicaciones periodísticas profesionales y su revelación a la comunidad científica, nos permitirá abrir nuevas vías de estudio para mejorar sustancialmente nuestros conocimientos sobre la profesión en los últimos siglos. Para ello, se ofrece en el presente trabajo una guía metodológica y un modelo para el estudio de las revistas enfermeras y de las ramas auxiliares sanitarias, facilitando la labor de los historiadores interesados en esta temática.

Agradecimientos: "A Francisco Herrera Rodríguez por sus orientaciones para la realización de este trabajo y por su entusiasmo efusivo hacia el estudio de la Historia de la Enfermería”.

\section{BIBLIOGRAFÍA}

- Aguirre Rojas, C.A. (2007). Antimanual del mal historiador. Barcelona: Ediciones de Intervención Cultura.

- Álvarez Nebreda, C.C. (2008). Catálogo bibliográfico de publicaciones enfermeras (1541-1978). Madrid: Colegio Oficial de Enfermería de Madrid.

- Álvarez Nebreda, C.C. (2010). Catálogo de publicaciones periódicas enfermeras (1864-1977). Madrid: Colegio Oficial de Enfermería de Madrid.

- Amezcua Martínez, M. (1994). El Colegio de Practicantes de Almería (1885-1945). Almería: Colegio Oficial de
Diplomados en Enfermería de Almería.

- Amezcua Martínez, M. (2000). La revista Índex de Enfermería: información bibliográfica, investigación y humanidades. Cultura de los Cuidados, (7-8), 68-74. Recuperado de http://culturacuidados.ua.es/enfermeria/article/ view/77/153.

- Aróstegui Sánchez, J. (1995). La investigación histórica: teoría y método. Barcelona: Crítica.

- Arratia Figueroa, A. (2005). Investigación y documentación histórica en enfermería. Texto \& Contexto Enfermagem, 14(4), 567-574. Recuperado de http://dx.doi. org/10.1590/S0104-07072005000400014.

- Ávila Olivares, J.A. (2012). Antecedentes corporativos a la colegiación en Alicante: Una carta reveladora. En C.C. Álvarez Nebred y F.J. Hernández Martín, (eds.) El asociacionismo en la enfermería y su influencia en el desarrollo de la profesión: 150 años de historia del Colegio de Enfermeras de Madrid (1862-2012). (pp.113-131). Madrid: Colegio Oficial de Enfermería de Madrid.

- Ávila Olivares, J.A. (2014). La protección social, factor determinante en el origen de la acción corporativa de los Auxiliares Sanitarios. Temperamentvm, (20). Recuperado de http://www.index-f.com/temperamentum/tn20/ t9807.php.

- Barrera del Barrio, C.(Ed.) (2000). El periodismo español en su historia. Ariel, Barcelona.

- Bernabeu Mestre, J., y Galiana Sánchez, M.E. (2009). Enfermería y exilio: el caso de las Visitadoras Sanitarias. Mètode, (61): 72-77. Recuperado de http://metode.cat/ es/Revistas/Monografics/Ciencia-y-exilio/Infermeria-iexili.

- Bernabeu Mestre, J., y Gascón Pérez, E. (1995). El papel de la enfermería en el desarrollo de la salud pública española (1923-1935): la visitadora sanitaria. Dynamis, 15, 151-176. Recuperado de en http://www.raco.cat/index.php/Dynamis/article/download/108730/149974.

- Bernabeu Mestre, J., y Gascón Pérez, E. (1999). Historia de la Enfermería de Salud Pública en España. Alicante: Publicaciones de la Universidad de Alicante.

- Cabeza Guillén, M.E., y Miqueo, C. (2013). La revista Súrgere (1959-1973), órgano de expresión profesional de las matronas españolas durante el franquismo. Tempera- 
mentvm, (18). Recuperado de http://www.index-f.com/ temperamentum/tn18/t9330.php.

- Callejas Chagoyen, I., y Miqueo, C. (2014). Conocimiento quirúrgico enfermero en la revista El Practicante (1885). Temperamentvm, (19). Recuperado de en http:// www.index-f.com/temperamentum/tn19/t9542.php.

- Calvo Calvo, M.A. (2005). Divulgación científica de la Historia de la Enfermería. Temperamentvm, (1). Recuperado de http://www.index-f.com/ temperamentum/1revista/a0105.php. Consultado el 12 de diciembre de 2014.

- Calvo Calvo, M.A. (2014). La reacción de los practicantes en Medicina y Cirugía frente a la creación del título de Enfermera en 1915. Dynamis, 34(2), 425446. Recuperado de http://dx.doi.org/10.4321/S021195362014000200008. Consultado el 12 de diciembre de 2014.

- Chamorro Rebollo, E. (2011). Análisis de las portadas de la revista ¡Firmes!: Revista de las Sanitarias Españolas de Salus Infirmorum. Temperamentvm, (13). Recuperado de http://www.index-f.com/temperamentum/tn13/ t7636.php. Consultado el 12 de diciembre de 2014.

- Córdoba Mariscal, M.A. (1994). El asociacionismo profesional a través de la revista Enfermeras (1950-1951). Jano, 47(1098), 81-86.

- Córdoba Mariscal, M.A., y Herrera Rodríguez, F. (1992). Una revista catalana de enfermería con proyección hispanoamericana (1950-1951). Anales de la Real Academia de Medicina y Cirugía de Cádiz, 28(1): 359-367.

- Expósito González, R. (2009a). Historia de la prensa profesional de los ministrantes y practicantes en España en el siglo XIX. Cultura de los Cuidados, 13(26): 12-21. Recuperado de http://culturacuidados.ua.es/enfermeria/ article/view/343/685. Consultado el 12 de diciembre de 2014.

- Expósito González, R. (2009b). La prensa profesional de los ministrantes y practicantes en España en el siglo XIX. Enfermería de Ciudad Real, (54): 30-33.

- Expósito González, R. (2013a). Apuntes para la Historia de la Organización Colegial de Enfermería en La Rioja (1884-1940). Crónicas de Enfermería, (32): 4-8.

- Expósito González, R. (2013b). Organización y lucha por el reconocimiento de los Colegios de Practicantes. En C.C. Álvarez Nebreda y F.J. Hernández Martín (Eds.), El asociacionismo en la enfermería y su influencia en el desarrollo de la profesión: 150 años de historia del Colegio de Enfermeras de Madrid (1862-2012 (pp.113-131). Madrid: Colegio Oficial de Enfermería de Madrid.

- Febvre, L. (1974). Combates por la historia. Barcelona: Ariel.

- Gálvez Toro, A., Hueso Montoro, C., Amezcua Martínez, M. (2005). Revistas internacionales de enfermería: comunidad científica hispanoportuguesa (año 2002). Índex de Enfermería, 14(50), 73-77.

- García Fernández, F.P., y Rodríguez Torres, M.C., Corres Urrechu, J.M. (1996). La "Revista del Practicante" (1945-1946): un bienio de lucidez. Índex de Enfermería, 5(18), 20-24.

- García Hernández, I., y Gálvez Toro, A. (2002a). La publicidad en revistas de enfermería españolas. Cultura de los Cuidados, 6(11), 80-86.

- García Hernández, I., y Gálvez Toro, A. (2002b). La publicidad en revistas de enfermería españolas II. Cultura de los Cuidados, 6(12), 100-102.

- García Martínez, A.C. (2013). Las publicaciones especializadas de los practicantes: Un agente de cambio profesional (1857-1936). En: C.C. Álvarez Nebreda y F.J. Hernández Martín (Eds.), El asociacionismo en la enfermería y su influencia en el desarrollo de la profesión: 150 años de historia del Colegio de Enfermeras de Madrid (18622012) (pp.91-112). Madrid: Colegio Oficial de Enfermería de Madrid.

- García Martínez, M.J., y Espina Gutiérrez, M.D. (19981999). Notas biográficas sobre Dña. Rosalía Robles Cerdán, fundadora-directora de la revista "La Matrona Hispalense”. Híades, (5-6), 327-332.

- García Menéndez, L. (1993). El "Boletín de Medicina, cirugía y farmacia" (1834-1839): una etapa trascendental en el periodismo científico español. Valladolid: Secretariado de Publicaciones de la Universidad de Valladolid.

- Gascón Pérez, E., y Bernabeu Mestre, J., Galiana Sánchez, M.E. (2002). La acción social de las visitadoras e instructoras sanitarias. Trabajo social y salud, (43), 149-176.

- Germán Bes, C. (2008). Historia de la Enfermería Comu- 
nitaria I. De enfermeras visitadoras a instructoras sanitarias. Temperamentvm, (8). Recuperado de http://www. index-f.com/temperamentum/tn8/t0608.php.

- González Canalejo, C., y Márquez Membrive, J. (2000). ¿Hacia dónde va la Historia de la Enfermería? Cultura de los Cuidados, 4(7-8), 27-34.

- Granero Molina, J., Fernández Sola, C., y Muñoz Ronda, F.J., Heredia Berciano, M., Muñoz París, M.J., y Fernández Miranda, E. (2002). La revista "El Practicante Almeriense". Cultura de los Cuidados, 6(12): 13-22.

- Heierle Valero, C. (2009). La imagen de la enfermera a través de los medios de comunicación de masas: la prensa escrita. Índex de Enfermería, 18(2), 95-98.

- Hernández Martín, F.J. (2008). Historiografía de la enfermería en España: la historia de na pequeña-gran familia. Cultura de los Cuidados, 12(24), 35-39.

- Hernández Sandoica, E. (2004). Tendencias historiográficas actuales: escribir historia hoy. Madrid: Akal.

- Herrera Rodríguez, F. (1989). Reflexiones sobre fuentes documentales para la investigación histórica de la Enfermería. Revista de Humanidades de Jerez, (1), 63-67.

- Herrera Rodríguez, F. (1993). Estudios sobre las profesiones auxiliares sanitarias en Cádiz (siglos XIX y XX). Anales de la Universidad de Cádiz, (9-10), 213-268. Recuperado de http://hdl.handle.net/10498/11439. Consultado el 12 de diciembre de 2014.

- Herrera Rodríguez, F. (1995). Un periódico aragonés: El Practicante (1885). Híades, (2): 153-167.

- Herrera Rodríguez, F. (2003). Nota sobre la Historia de la Enfermería en España (1977-2002). Llull, 26: 157173. Recuperado de http://dialnet.unirioja.es/descarga/ articulo/831836.pdf. Consultado el 12 de diciembre de 2014.

- Herrera Rodríguez, F. (2008). Archivos y hemerotecas: espacios para la investigación de la Historia de la Enfermería. Híades, (10), 71-110.

- Herrera Rodríguez, F., y Cabrera Afonso, J.R. (2001). El Colegio Oficial de Médicos de la Provincia de Cádiz (1898-1936). En J.R. Cabrera Afonso y F. Herrera Rodríguez, (Eds.) El Excmo. Colegio Oficial de Médicos de la Provincia de Cádiz en el siglo XX. Conmemoración de su Centenario (1901-2001) (pp.63-161). Cádiz: Excmo. Co- legio Oficial de Médicos de la Provincia de Cádiz.

- Herrera Rodríguez, F., y Lasarte Calderay, J.E. (1996). La prensa andaluza de los practicantes y matronas (19161939). En I Congreso Nacional de Historia de la Enfermería (pp.169-174). Madrid: Universidad Complutense de Madrid.

- Itayra Padilla, M., Borenstein, M., y Guedes, J. (2007). Investigación histórica en la enfermería: posibilidades y metodología. Temperamentvm, (5). Recuperado de http://www.index-f.com/temperamentum/tn5/t1207. php. Consultado el 12 de diciembre de 2014.

- Kayser, J. (1966). El periódico: Estudios de morfología, de metodología y prensa comparada (3a edición). Quito: CIESPAL. Recuperado de http://repositorio.ciespal. org:8080/bitstream/123456789/168/2/CIESPAL.pdf. Consultado el 12 de diciembre de 2014.

- Larra Cerezo, A. (1905). Historia resumida del periodismo médico en España. Madrid: Imprenta de Ricardo Rojas.

- Lasarte Calderay, J.E. (1993). La prensa de las profesiones auxiliares sanitarias en Andalucía. Estudio socio-profesional (1916-1939).Cádiz: Universidad de Cádiz. Recuperado de http://hdl.handle.net/10498/15637. Consultado el 12 de diciembre de 2014 .

- Lasarte Calderay, J.E. (2000). Fuentes iconográficas de enfermería en la revista Nuevo Mundo (1896-1930). Híades, (7), 11-54.

- López Piñero, J.M., y López Terrada, M.L. (1980). Las etapas históricas del periodismo médico en España. Estudio bibliométrico. En A. Albarracín Teulón., J.M. López Piñero., y Sánchez Granjel, L. (Eds.) Medicina e Historia (pp.163-191). Madrid: Editorial de la Universidad Complutense.

- López Piñero, J.M., y López Terrada, M.L. (1990). Bibliographia Médica Hispánica 1475-1950. Volumen VIII: Revistas, 1736-1950. Valencia: Instituto de Estudios Documentales e Históricos sobre la Ciencia. CSIC.

- Marín Paz, A.J. (2014). El Practicante Moderno (19031904) y la I Asamblea de Practicantes de España: el asociacionismo del colectivo en el periodo regeneracionista. Temperamentvm, (20). Recuperado de http://www. index-f.com/temperamentum/tn20/t9976.php. Consultado el 08 de enero de 2015. 
- Martínez Sánchez, J. (1901). La Odontología. Índice bibliométrico: bibliografía dental al terminar el siglo XX. Madrid: Imprenta de la Odontología.

- Moral de Calatrava, P., y Hernández Conesa, J.M. (2007). Investigación, teoría e Historia de la Enfermería: Categorías Históricas para la construcción de la Identidad enfermera. Temperamentvm, (6). Recuperado de http:// www.index-f.com/temperamentum/tn6/t1107.php. Consultado el 12 de diciembre de 2014.

- Ortega y Gasset, J. (1984). Historia como sistema y otros ensayos filosóficos. Madrid:Editorial Sarpe.

- Pedraz Marcos, A., Ramírez Schacke, M., Oter Quintana, C., Palmar Santos, A., y Martín Robledo, E. (2007). La Visitadora Sanitaria. Órgano oficial de la Asociación Profesional de Visitadoras Sanitarias. Temperamentvm, (6). Recuperado de http://www.index-f.com/temperamentum/tn6/t6466.php. Consultado el 12 de diciembre de 2014 .

- Porto, F., y Franco Santos, T.C. (2009). Propagandas de remédio na imprensa ilustrada e a imagem da enfermeira brasileira (1920-1925). Revista de Escola de Enfermagem da USP, 44(3), 819-826. Recuperado de http://dx.doi. org/10.1590/S0080-62342010000300038. Consultado el 12 de diciembre de 2014.

- Riera Palmero, J. (Introducción, edición e índices) (1978). Francisco Méndez Álvaro. Historia del periodismo médico y farmacéutico en España. Valladolid: Universidad de Valladolid.

- Rodríguez García, A.R. (1994). Nueva profesión de enfermeras. La noticia: 1915. JANO, 47(1.098), 59-64.

- Romano, D. (1983). Elementos y técnicas del trabajo científico. Barcelona: Editorial Teide.

- Salmon, P. (1978). Historia y crítica. Introducción a la metodología histórica. Barcelona: Editorial Teide.

- Santo Tomás Pérez, M. (2006). La investigación en la Historia de la Enfermería: líneas de futuro. Temperamentvm, (3). Recuperado de http://www.index-f.com/ temperamentum/tn3/t0106.php. Consultado el 12 de diciembre de 2014.

- Santo Tomás Pérez, M. (2013). Pugna por el reconocimiento universitario y su especialización. En C.C. Álvarez Nebreda y F.J. Hernández Martín (Eds.), El asocia- cionismo en la enfermería y su influencia en el desarrollo de la profesión: 150 años de historia del Colegio de Enfermeras de Madrid (1862-2012)(pp.297-331). Madrid: Colegio Oficial de Enfermería de Madrid.

- Sanz Hernández, M.A. (1995). Fuentes orales y documentales en la investigación social. Proyecto social: $R e$ vista de relaciones laborales, (3), 217-230.

- Siles González, J. (2001a). Los textos como fuente de conocimientos en ciencias sociales y enfermería. Una aplicación de la metodología cualitativa. Índex de Enfermería, 10(32-33), 28-34.

- Siles González, J. (2001b). Los textos como fuente de conocimientos en ciencias sociales y enfermería (y 2). Una aplicación de la metodología cualitativa. Índex de Enfermería, 10(34), 33-36.

- Siles González, J. (2004). La construcción social de la Historia de la Enfermería. Índex de Enfermería, 13(47), 7-10.

- Siles González, J. (2011). Historia de la Enfermería. Madrid: DAE.

- Thuillier, G., Tulard, J (1988). Cómo preparar un trabajo de historia. Métodos y técnicas.

Barcelona: Editorial Oikos-Tau.

- Tuñón de Lara, M. (1985). Por qué la Historia. Barcelona: Aula Abierta Salvat.

- Tuñón de Lara, M. (2010). Metodología de la historia social de España (6 edición). Madrid: Siglo XXI de España Editores.

- Valle Racero, J.I. (2000). El trabajo con documentos. Una aproximación a la historia de las mentalidades. Índex de Enfermería, 9(31), 25-27.

- Velandia Mora, A.L. (2011). Fundamentos teórico metodológicos de investigación en historia de la enfermería. Cultura de los Cuidados, 15(31): 118-126. 